\title{
Stimulated Thermal Scattering Induced by Two-Photon Absorption and Experimental Observation of the Genuine Stimulated Brillouin Scattering in the Near-Ultraviolet Region
}

\author{
Vladimir Boris Karpov \\ Coherent and Nonlinear Optics Department, A.M.Prokhorov General Physics Institute, Russian Academy of Sciences, Moscow, Russia
}

Email address:

karpov@kapella.gpi.ru (V. B. Karpov)

To cite this article:

Vladimir Boris Karpov. Stimulated Thermal Scattering Induced by Two-Photon Absorption and Experimental Observation of the Genuine Stimulated Brillouin Scattering in the Near-Ultraviolet Region. American Journal of Optics and Photonics. Vol. 3, No. 5, 2015, pp. 65-79. doi: 10.11648/j.ajop.20150305.13

\begin{abstract}
Part of the experimental measurements of the frequency shift and the phase-conjugation (PC) fidelity gained from previous studies of stimulated scattering (SS) of nanosecond ( $5 \sim 10 \mathrm{~ns}$ ) near-ultraviolet (UV) $(\lambda=193 \sim 351 \mathrm{~nm})$ laser pulses in liquids (hexane, heptane, and others) are found to disagree with the theory of SS, which takes into account only the linear (single-photon) light absorption. To resolve the inconsistency, SS of XeCl excimer laser radiation $(\lambda=308 \mathrm{~nm})$ with the duration of $8 \mathrm{~ns}$ in liquid hexane is investigated experimentally. A theoretical analysis of the results obtained revealed three new nonlinear optical phenomena induced by the heating due to two-photon absorption: stimulated thermal scattering (two-photon STS-2), phase mismatch for stimulated Brillouin scattering (SBS), and phase self-modulation. Experimental SS spectrum contains two new lines - a previously unknown two-photon STS-2 line and a genuine SBS line newly discovered in the near-UV region.
\end{abstract}

Keywords: Nonlinear Optics, Stimulated Brillouin Scattering (SBS), Stimulated Temperature Scattering (STS),

Two-Photon Heating, Near Ultraviolet Radiation, Excimer Lasers

\section{Introduction}

Stimulated scattering (SS) is widely used in scientific research and practical applications [1 - 5]. This motivates studies of the physical mechanisms responsible for SS in various spectral regions. An important application is phase conjugation (PC) via stimulated backscattering (PC via $\mathrm{SS}$ ) [6]. Various SS mechanisms have specific characteristics (frequency shift, decay time, etc.) that manifest themselves in the PC mirrors properties [4].

Detailed experimental studies of SS have been conducted only in the near-infrared (near-IR) region. For such experiments the pump radiation must have both high power and narrow bandwidth. The first sources of this kind were Q-switched single-mode ruby $(\lambda=0.69 \mu \mathrm{m})$ and Nd:glass $(\lambda=1.06 \mu \mathrm{m})$ lasers. For weak linear absorption, stimulated Brillouin scattering (SBS) and stimulated Raman scattering (SRS) were usually observed, for stronger linear absorption stimulated thermal scattering caused by the heating due to linear absorption (linear STS-2).

In the first experimental studies of SBS for the near-IR pump radiation [7 - 10] the unshifted spectral components were supposed to be the pump spectral lines. But under the experimental conditions of [7 - 10] the pump spectral lines could not be distinguished from slightly-shifted components corresponding, for instance, to linear STS-2.

Multiphoton absorption could not influence the experiments because 5 $\sim 10$ near-IR photons with energies $1 \sim 2 \mathrm{eV}$ would be required to obtain the nearest electron resonance with an energy about $10 \mathrm{eV}$.

Theoretical studies of SS have mostly relied on experimental results obtained for the near-IR region, and the modern SS theory applies only to linearly absorbing media [1 $-5,11,12]$. The theory of stimulated thermal scattering (STS) proposed in $[13,14]$ incorporates the linear absorption only.

Reliable near-ultraviolet (near-UV) radiation sources 
(discharge $\mathrm{XeF}, \mathrm{XeCl}, \mathrm{KrF}$, and $\mathrm{ArF}$ excimer lasers) became available considerably later than the solid-state near-IR lasers. When experimentalists in 1980-s had to deal with the PC via SS driven by excimer laser beams [15 - 22], the SS theory developed for the near-IR was applied to the near-UV spectral region.

\section{Previous Studies of Stimulated Scattering and Phase Conjugation in the Near-Ultraviolet Region}

The first studies concerning the PC via SS in liquids (hexane, heptane, and others) were reported in [15] (XeF, $\lambda=351 \mathrm{~nm}),[18](\mathrm{XeCl}, \lambda=308 \mathrm{~nm}),[16,19](\mathrm{KrF}$, $\lambda=248 \mathrm{~nm})$, and [17] (ArF, $\lambda=193 \mathrm{~nm})$. In the above and subsequent $[20,21]$ studies, experimental SS spectrum comprised a single shifted line, which was attributed to "SBS". Note that the frequency shift of the "SBS" line in [17 - 19] was comparable to the laser system spectral resolution.

The reflection coefficient of the "Brillouin" mirror measured in [19] gradually decreased with increasing pump intensity $I_{L}$ from $25 \%$ at the threshold $\left(I_{L}^{T H R} \approx 10^{10} \mathrm{~W} / \mathrm{cm}^{2}\right)$ to $10 \%$ at $I_{L} \approx 10^{11} \mathrm{~W} / \mathrm{cm}^{2}$. Note that in this experiment the decrease in the reflection coefficient was due to the decrease in the PC fidelity for the "Brillouin" mirror.

In $[15,16,18,20]$ the PC fidelity for the "Brillouin" mirror was found to degrade with increasing linear absorption coefficient $\alpha$ of the nonlinear liquid.

In [22] experimental SS spectrum obtained in hexane by using $\mathrm{KrF}$ laser radiation also comprised a single line, which was attributed to the "linear STS-2".

The linear absorption coefficient varied from $\alpha=0.02$ to $0.1 \mathrm{~cm}^{-1}$ in the studies of "SBS" [15 - 21] and amounted to $\alpha=0.22 \mathrm{~cm}^{-1}$ in the investigation of "linear STS-2" [22].

In order to increase the pump intensities in [15 - 22], the amplified laser beams were focused by lenses with focal lengths $F=5 \sim 10 \mathrm{~cm}$. The focal caustics corresponded to the volumes of the nonlinear interaction, and their lengths (see Section 6.2) were $L \leq 0.1 \mathrm{~cm}$. A considerable intensity attenuation due to the linear absorption over such distance occurs when $\alpha>1 \mathrm{~cm}^{-1}$, which exceeds the values mentioned above.

The pump pulse durations in [15-22] were $\tau_{0}=5 \sim 10 \mathrm{~ns}$.

Table 1 summarizes the results gained from the previous studies of stimulated backscattering in hexane for the near-UV pump radiation. The first column presents a reference to the previous study; the second and third columns - the pump wavelength $\lambda$ and intensity $I_{L}$; the fourth column - the experimentally measured frequency shift $\Omega$; the last column - the physical mechanism, which, the authors of the reference believe to be responsible for the observed SS.

\section{Inconsistency Between Previous Experimental Results and the Existing Theory of Stimulated Scattering}

\section{1. "SBS" Frequency Shift}

No one in the previous studies of "SBS" [15 - 21] calculated the theoretically predicted frequency shift to compare it with the experimentally measured one.

In accordance with the existing theory [1 - 5], the SBS frequency shift $\Omega_{B}$ depends on the pump frequency $\omega_{L}$ (the pump wavelength $\lambda$ ) and the scattering angle $\theta$ (see Eq. (13) below). Table 2 shows the values of $\Omega_{B}$ calculated for hexane, $\theta=\pi$, and the pump wavelengths listed in Table 1. It is clear that for the same $\lambda$ the predicted values (Table 2) disagree with the measured values attributed to "SBS" (Table 1). The disagreement, which is too large to be entirely caused by experimental errors, emerges for the pump wavelengths ranging from $\lambda=193$ to $351 \mathrm{~nm}$.

Table 1. Previous studies of the stimulated backscattering in hexane for the near-UV pump radiation generated by $\mathrm{ArF}$ (193 nm), $\mathrm{KrF}$ (248 nm), XeCl $(308 \mathrm{~nm})$, and $\mathrm{XeF}(351 \mathrm{~nm})$ excimer lasers.

\begin{tabular}{|c|c|c|c|c|}
\hline Reference & $\begin{array}{l}\text { Pump } \\
\text { wavelength } \\
\lambda, n m\end{array}$ & $\begin{array}{l}\text { Pump } \\
\text { intensity } \\
I_{L}, W / \mathrm{cm}^{2}\end{array}$ & $\begin{array}{l}\text { Experimentally } \\
\text { measured } \\
\text { frequency shift } \\
\text { in hexane } \\
\Omega, \mathrm{cm}^{-1}\end{array}$ & $\begin{array}{l}\text { Physical } \\
\text { mechanism } \\
\text { of SS } \\
\text { suggested } \\
\text { by authors } \\
\text { of the } \\
\text { reference }\end{array}$ \\
\hline [17] & 193 & $\approx 10^{10}$ & $\approx 0.2$ & SBS \\
\hline [16] & 248 & $>2 \times 10^{10}$ & $\approx 0.1$ & SBS \\
\hline [19] & 248 & $>10^{10}$ & $\approx 0.1$ & SBS \\
\hline [18] & 308 & $>10^{11}$ & $<0.15$ & SBS \\
\hline [21] & 308 & $>10^{12}$ & 0.24 & SBS \\
\hline [15] & 351 & $>5 \times 10^{9}$ & 0.2 & SBS \\
\hline [22] & 248 & $>10^{11}$ & $<0.02$ & $\begin{array}{l}\text { Linear } \\
\text { STS-2 }\end{array}$ \\
\hline
\end{tabular}

Table 2. Theoretically predicted backward $(\theta=\pi)$ SBS frequency shift $\Omega_{B}$ in hexane for the pump wavelengths listed in Table 1.

\begin{tabular}{ll}
\hline Pump wavelength $\lambda, \mathrm{nm}$ & $\begin{array}{l}\text { Theoretically predicted SBS frequency } \\
\text { shift in hexane } \Omega_{B}, \mathrm{~cm}^{-1}\end{array}$ \\
\hline 193 & 0.49 \\
248 & 0.41 \\
308 & 0.33 \\
351 & 0.30 \\
\hline
\end{tabular}

\subsection{Phase Conjugation Fidelity for "Brillouin" Mirror}

The decreasing PC fidelity for increasing above the threshold pump intensity detected in [19] is not a dependence of SBS, because, in accordance with the existing theory [4, 11], the strong pump saturation improves the discrimination of noises. 


\subsection{Emergence of "Linear STS-2" Component and Disappearance of "SBS" Component}

Emergence of the "linear STS-2" component in [22] was explained by the increase in the linear absorption coefficient from $\alpha=0.02 \sim 0.1 \mathrm{~cm}^{-1}$ mentioned in [15 - 21], to $\alpha=0.22 \mathrm{~cm}^{-1}$. But it was not explained how such increase in $\alpha$ could cause the suppression of "SBS" in [22]. Indeed, the "SBS" component has been observed in [15 - 21] practically for the same experimental conditions with the exception of $\alpha$.

\section{Experimental Setup}

The composite problem encompasses both the temporal and the spatial behavior manifested in the frequency shift and the PC fidelity, respectively. The abnormal "SBS" was detected in a variety of studies conducted by a number of laboratories for nearly a decade [15 - 21], which rules out accidental experimental errors. This problem may be resolved by examining the fundamental physical mechanisms responsible for SS.

To investigate the physical mechanisms responsible for stimulated backscattering of nanosecond near-UV pulses, we constructed experimental setup (Fig. 1) based on a couple of commercially available ELI-91 $\mathrm{XeCl}$ excimer lasers $(\lambda=308 \mathrm{~nm})$. The setup was designed as a master-oscillator-power-amplifier (MOPA) system. Hexane was used as the nonlinear liquid.

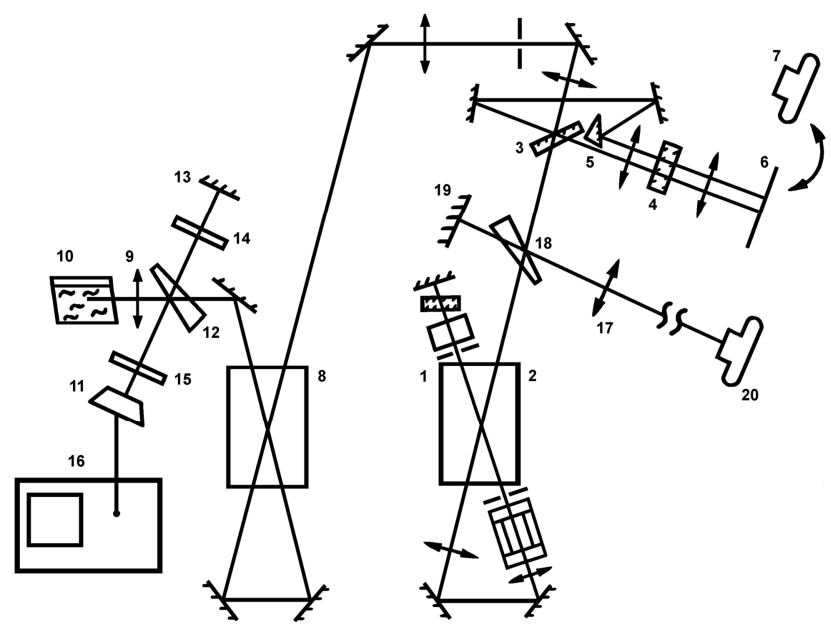

Fig. 1. Experimental setup for the investigation of the stimulated backscattering of a XeCl excimer laser beam in hexane: (1) the master oscillator and (2) the preamplifier based on the first ELI-91 XeCl laser; (3, 12 , 18) the beamsplitters; (4) the Fabry-Perot etalon and (5) the prism of the spectrum analyzer; (6) the luminescent screen; $(7,20)$ the cameras; (8) the two-passes amplifier based on the second ELI-91 XeCl laser; (9) the lens with one of three possible focal lengths $F=11$, or 50 , or $100 \mathrm{~cm}$; (10) the cell filled with hexane; (11) the FEK-29KPU photodiode and (16) the S7-19 oscilloscope of the time-domain analyzer; $(13,19)$ the mirrors; $(14,15)$ the neutral filters; (17) the divergence analyzer lens with the focal length $f_{1}=3 \mathrm{~m}$

\subsection{Master Oscillator}

The master oscillator (MO) generates a weak narrow-divergence, narrow-bandwidth beam

The diffraction-limited divergence was created by two apertures of radius $r_{0}=0.8 \mathrm{~mm}$ mounted on both sides of the MO active cell.

The bandwidth was narrowed by means of an MO cavity with a nontransparent mirror at one end and a mode selector at the other. The selector operates as an interference filter with a frequency-dependent reflection coefficient. Originally [23], a selector of this kind was used in a Nd:glass laser characterized by the spontaneous lifetime close to $1 \mu \mathrm{s}$. The spontaneous lifetime for a $\mathrm{XeCl}$ laser is about $20 n s$. For a $1 m$ - long cavity, the number of roundtrips is 6 , which is not sufficient to select a single longitudinal mode. The optical system proposed in [23] is useless when applied to the $\mathrm{XeCl}$ laser. However, a single longitudinal mode was selected by using an extra Fabry-Perot etalon placed inside the MO cavity.

The MO output characteristics are presented in the third column of Table 3; the characteristics of a commercially available ELI-91 laser are listed in the second column.

Table 3. Laser parameters.

\begin{tabular}{llll}
\hline Parameter & $\begin{array}{l}\text { Commercially } \\
\text { available } \\
\text { ELI-91 laser }\end{array}$ & $\begin{array}{l}\text { Master } \\
\text { oscillator }\end{array}$ & $\begin{array}{l}\text { Amplified } \\
\text { beam }\end{array}$ \\
\hline $\begin{array}{l}\text { Bandwidth, } \mathrm{cm}^{-1} \\
\text { Aperture }\end{array}$ & 15 & $5 \times 10^{-3}$ & $5 \times 10^{-3}$ \\
$\begin{array}{l}\text { Divergence, rad } \\
\text { Pulse duration }\end{array}$ & $10 \mathrm{~mm} \times 20 \mathrm{~mm}$ & $\varnothing 1.6 \mathrm{~mm}$ & $8 \mathrm{~mm} \times 10 \mathrm{~mm}$ \\
$\begin{array}{l}\text { (FWHM), ns } \\
\text { Pulse energy, } \mathrm{mJ}\end{array}$ & 20 & $6 \times 10^{-4}$ & $3 \times 10^{-4}$ \\
Wavelength, nm & 50 & 8 & 8 \\
\hline
\end{tabular}

\subsection{Experimental Technique}

The beam generated by MO 1 was expanded by a telescope and preamplified in the same active volume 2 of the first ELI-91 XeCl laser to approximately $1 \mathrm{~mJ}$. Upon going through two-passes amplifier 8 based on the second ELI-91 $\mathrm{XeCl}$ laser, the pulse had a nearly Gaussian temporal shape with the duration (FWHM) about $8 n s$, energy about $3 \mathrm{~mJ}$, and bandwidth about $5 \times 10^{-3} \mathrm{~cm}^{-1}$. Characteristics of the amplified beam are specified in the last column of Table 3 .

The amplified beam was focused by lens 9 into cell 10 filled with hexane. The nonlinear interaction took place in the focal caustic of lens 9 . The backscattered wave traveled back through the system and was amplified in 8 .

Beamsplitter 12 was used to guide both forward- and backward-propagating beams into the time-domain analyzer. The temporal power profiles were measured by means of FEK-29KPU photodiode 11 (the resolution being equal to 200 ps ) and S7-19 oscilloscope 16 (the bandwidth being equal to $5 \mathrm{GHz}$ ). The phase-conjugation mirror reflectivity was determined as the backscattered beam to the amplified beam peak power ratio.

Beamsplitter 3 was used to guide both forward- and backward-propagating beams into the spectrum analyzer (Fabry-Perot etalon 4 of a variable dispersion-free region combined with prism 5), which produced the composite 
temporal spectrum of both the pump wave and the stimulated backscattered wave simultaneously on luminescent screen 6 or camera 7. This facilitated the measurement of the relative frequency shift. The temporal spectra were not changed by amplification in 8 .

Beamsplitter 18 was used to guide both forward- and backward-propagating beams into the divergence analyzer. For each beam, camera 20 created a photographic image of the focal spot produced by lens 17 with the focal length $f_{1}=3 \mathrm{~m}$. This photographic image carries only the amplitude information. Since the phase modulation gets transformed into the amplitude one in the focal region as in the far field, the beam divergence $\theta_{L}$ can be calculated from the relation $\theta_{L} \approx \frac{d}{f_{1}}$, where $d$ is the focal spot diameter. In order to measure the amplified beam divergence the divergence analyzer was placed into the output of amplifier 8 .

\subsection{Nonlinear Liquids and Cells}

In the previous experiments [15 - 22], the amplified beams were focused by lenses with the focal lengths $F=5 \sim 10 \mathrm{~cm}$ into about $5 \mathrm{~cm}$ - long cells filled with nonlinear liquids. Organic solvents utilized in the UV chromatography, such as hexane, heptane, ethanol were used as the nonlinear liquids. Similar results were obtained for different liquids. Since hexane $\left(\mathrm{C}_{6} \mathrm{H}_{14}\right)$ was investigated in almost all of these studies (see Table 1), we also chose it.

In our experiments, the amplified beam was focused by lens 9 into the centre of cylindrical glass cell 10 with internal diameter $40 \mathrm{~mm}$ filled with hexane. We used $5 \mathrm{~cm}$ - long cells in combination with lens 9 having $F=11 \mathrm{~cm}$ and $30 \mathrm{~cm}$ - long cells in combination with lens 9 having $F=50,100 \mathrm{~cm}$. Each cell was sealed by a couple of fused-silica input/output windows sloped at the angle of $5^{0}$ to avoid parasitic reflections.

We used the HPLC-grade ("chromatography-grade") hexane produced by Oldrich Chemical Co., Milwaukee, WI, and the "chemically pure" hexane from Russian suppliers. Their linear absorption coefficients measured at $\lambda=308 \mathrm{~nm}$ were $\alpha=(0.01 \pm 0.003) \mathrm{cm}^{-1}$ and $\alpha=(0.046 \pm 0.003) \mathrm{cm}^{-1}$, respectively.

We added extremely small amounts of acetone to hexane to increase slightly the linear absorption coefficient, so that cells used in our experiments were characterized by $\alpha=0.01,0.046,0.08,0.17 \mathrm{~cm}^{-1}$ at $\lambda=308 \mathrm{~nm}$.

The refractive index of hexane at $\lambda=308 \mathrm{~nm}$ and its temperature derivative at constant pressure are [2] $n \approx 1.4$, $\left(\frac{\partial n}{\partial T}\right)_{p}=-53 \times 10^{-5} K^{-1}$. The corresponding permittivity and

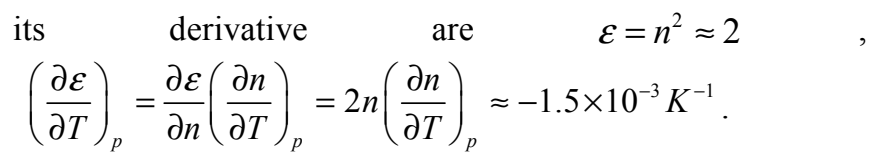

\section{Experimental Results}

\subsection{Experiment 1: The Dependence of the Temporal Stimulated Backscatter Spectrum on the Pump Intensity}

When constant parameters of the amplified beam (see Table 3) are maintained, the pump intensity in cell 10 (see Fig. 1) can be varied by means of neutral filters or by changing focal length of lens 9. The SS was close to its threshold for any lens 9 employed. Therefore, we could not use neutral filters which reduce the amplified beam power. The pump intensity $I_{L}$ was varied only by changing the focal length $F$ of lens 9 .

Three different lenses 9 with $F=11,50$, and $100 \mathrm{~cm}$ were used. For each lens ( $F=$ const ), the composite temporal spectrum was obtained by the spectrum analyzer described above with Fabry-Perot etalon 4 having the dispersion-free region of $0.66 \mathrm{~cm}^{-1}$. Only the chromatography-grade hexane ( $\left.\alpha=0.01 \mathrm{~cm}^{-1}\right)$ was used.

Figure 2 shows three images corresponding to the three values of $F$. Each photography comprises two parts: the pump spectrum (right) and the stimulated backscatter spectrum (left). The stimulated backscatter spectrum has a single unshifted component when $F=11 \mathrm{~cm}$ (Fig. 2a); two components when $F=50 \mathrm{~cm}$ : an unshifted one and a Stokes one shifted by $0.33 \mathrm{~cm}^{-1}$ (Fig. 2b); and a single Stokes component shifted by $0.33 \mathrm{~cm}^{-1}$ when $F=100 \mathrm{~cm}$ (Fig. 2c).

\subsection{Experiment 2: The Dependence of the Temporal Stimulated Backscatter Spectrum on the Linear Absorption Coefficient}

We used all cells having $\alpha=0.01,0.046,0.08,0.17 \mathrm{~cm}^{-1}$. For each $\alpha=$ const we examined the dependence of the stimulated backscatter spectrum on the pump intensity, which was controlled by changing the focal length of lens 9 . Dependence on $F$ for $\alpha=0.046,0.08 \mathrm{~cm}^{-1}$ was similar to that for $\alpha=0.01 \mathrm{~cm}^{-1}$ illustrated by Figs. 2a - 2c. Each stimulated backscatter spectrum obtained for $\alpha=0.17 \mathrm{~cm}^{-1}$ contained a single unshifted component (as in Fig. 2a).

\subsection{Experiment 3: The Dependence of the Phase-Conjugation Fidelity on the Pump Intensity}

When the forward-propagating beam passes amplifier 8, its divergence increases by a factor of 3 . We measured the divergences of forward- and backward-propagating beams at the location of beamsplitter 18 with the help of the divergence analyzer described above. We examined the dependence of the backward-propagating beam divergence on the pump intensity, which was controlled by changing the focal length of lens 9 . We used only the chromatography-grade hexane ( $\alpha=0.01 \mathrm{~cm}^{-1}$ ) to avoid the thermal self-action caused by the linear absorption. The experiment provided the information on the phase-conjugation fidelity: when the PC fidelity is "high", the forward and the backward aberrations must 
compensate each other.

Figure 3 shows three photographic images created by camera 20. The resulting divergences are: $\theta_{L} \approx 3 \times 10^{-4} \mathrm{rad}$ for a forward-propagating beam (Fig. 3a); $\theta_{L} \approx 3 \times 10^{-4} \mathrm{rad}$ for a "high" phase-conjugation fidelity backscattered beam obtained with $F=100 \mathrm{~cm}$ (Fig. $3 b$ ); and $\theta_{L} \approx 2 \times 10^{-3} \mathrm{rad}$ for a "poor" phase-conjugation fidelity backscattered beam obtained with $F=11 \mathrm{~cm}$ (Fig. 3c).

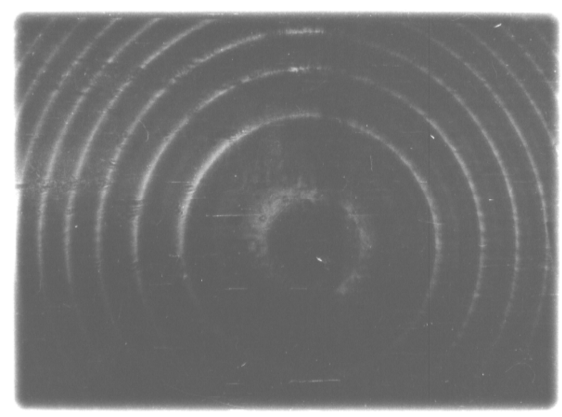

a

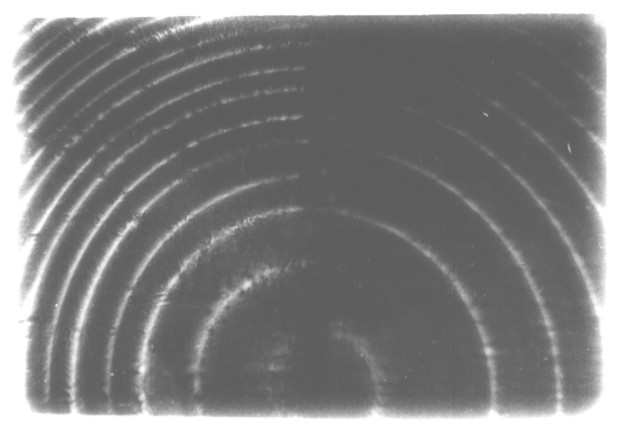

b

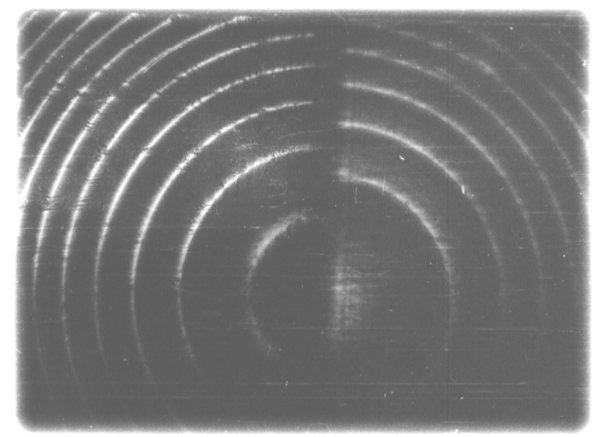

Fig. 2. Results of Experiments 1 and 2: three composite temporal spectra obtained by the spectrum analyzer with Fabry-Perot etalon 4 having the dispersion-free region of $0.66 \mathrm{~cm}^{-1}$. Each photography comprises two parts: the pump spectrum (right) and the stimulated backscatter spectrum (left) in hexane at a constant linear absorption coefficient $\alpha$ for three focal lengths of lens 9: $F=(a) 11,(b) 50,(c) 100 \mathrm{~cm}$. The dependence of the stimulated backscatter spectrum on $F$ is similar for $\alpha=0.01,0.046,0.08 \mathrm{~cm}^{-1}$.

\section{C}
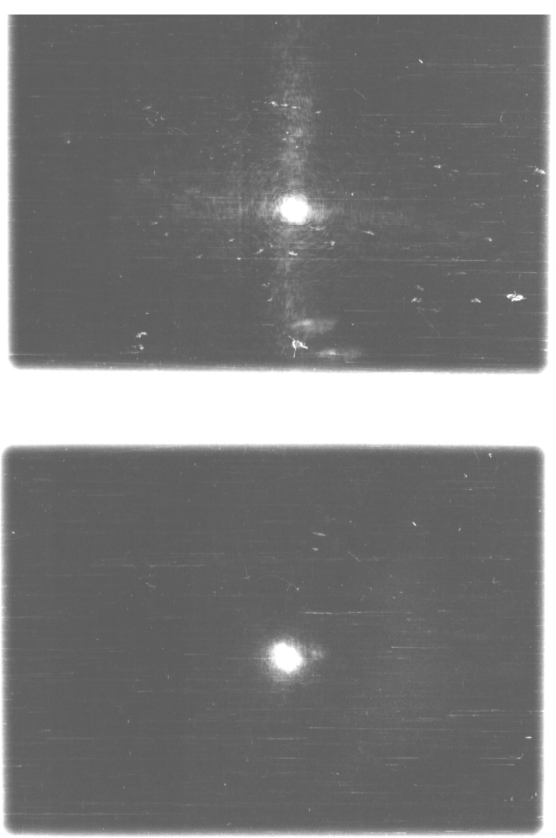

b

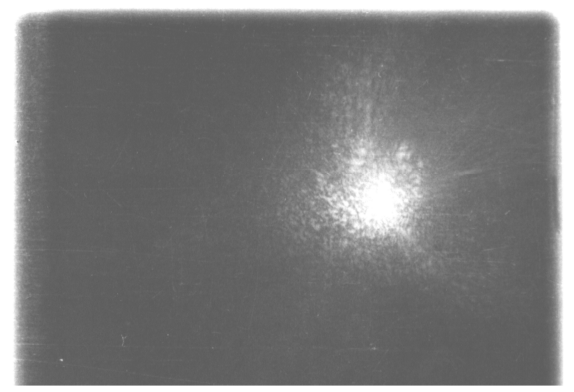

Fig. 3. Results of Experiment 3: three focal spots obtained by the divergence analyzer for (a) a forward-propagating beam; (b) a backward-propagating beam ( $F=100 \mathrm{~cm}$, "high" phase-conjugation fidelity); (c) a backward-propagating beam ( $F=11 \mathrm{~cm}$, “poor” phase-conjugation fidelity). Images (b) and (c) were obtained for hexane with $\alpha=0.01 \mathrm{~cm}^{-1}$.

\subsection{Experiment 4: The Temporal Power Profiles and the Phase-Conjugation Mirror Reflectivity}

The analysis of the temporal evolution of the pulses with the time-domain analyzer described above is important, because the nonlinear mirror may change their amplitudes, durations, and shapes. All the cells used had $\alpha=0.01,0.046,0.08,0.17 \mathrm{~cm}^{-1}$.

The backscattered temporal power profile was found to have a nearly Gaussian shape and the duration (FWHM) of approximately $7 n s$. The slightly shorter duration as compared to the pump one (see Table 3) can be attributed to the threshold nature of SS.

The phase-conjugation mirror reflectivity reached $20 \%$ for $\alpha=0.01 \mathrm{~cm}^{-1}$ and gradually decreased with increasing $\alpha$. The reflectivity increased as the focal caustic of lens 9 was shifted from the centre toward the input window of cell 10 . 


\section{Analysis and Interpretation of Experimental Results}

\subsection{Results of Experiments Which Require Theoretical Analysis}

Trends revealed in our Experiments 1 - 4 on stimulated backscattering in hexane for different $\alpha$ and $F$ that require further theoretical analysis can be summarized as follows.

1. The dependence of the backscatter spectrum on $F$ obtained for $\alpha=0.01 \sim 0.08 \mathrm{~cm}^{-1}$ :

(1.1) the presence of two components when $F=50 \mathrm{~cm}$ : an unshifted one (with the experimental error $0.02 \mathrm{~cm}^{-1}$ ) and the Stokes one shifted by $0.33 \mathrm{~cm}^{-1}$ (Fig. 2b);

(1.2) the disappearance of the unshifted component when $F$ is increased from 50 to $100 \mathrm{~cm}$ (Figs. 2b and 2c);

(1.3) the disappearance of the shifted component when $F$ is reduced from 50 to $11 \mathrm{~cm}$ (Figs. $2 \mathrm{a}$ and $2 \mathrm{~b}$ ).

2 . The dependence of the backscatter spectrum on variation from $\alpha=0.01 \sim 0.08$ to $\alpha=0.17 \mathrm{~cm}^{-1}$ :

(2.1) the disappearance of the shifted component when $F=50,100 \mathrm{~cm}$;

(2.2) the appearance of an unshifted component when $F=100 \mathrm{~cm}$.

3. The substantial decrease in the phase-conjugation fidelity obtained when $\alpha=0.01 \mathrm{~cm}^{-1}$ is maintained at a constant level and $F$ is reduced from 100 to $11 \mathrm{~cm}$ (Figs. 3b and $3 \mathrm{c})$.

\subsection{The Specific Features of the Stimulated Scattering in Our Experiments}

At the initial stage of the SS process, a weak scattered wave is generated as a result of the spontaneous scattering of the pump wave. In the resulting steady state without considerable pump saturation, the scattered wave intensity $I_{S}$ is expressed as $[1-5]$ :

$$
I_{S}(L)=I_{S}(0) \exp \left(I_{L} G L\right)
$$

where $I_{S}(0)$ is the spontaneously scattered intensity, $I_{L}$ is the pump intensity, $G$ is the gain factor, and $L$ is the nonlinear interaction length. The threshold condition for SS is

$$
\left(I_{L} G L\right)^{T H R} \approx 30
$$

If the total gain $\left(I_{L} G L\right)$ for a certain SS mechanism is below the threshold (2), the mechanism does not contribute to the overall SS pattern observed in an experiment.

Unfortunately, the 3-D calculations of the real beam focal caustic are problematic even for vacuum. For organic liquids, only rough approximations can be used. Fortunately, in contrast to the near-IR, for the near-UV due to the fluorescence of hexane a blue track of the focused laser beam was simply observed through a thin transparent glass wall of the cell (of course, in the dark lab). The focal caustic geometry was measured and successively compared with the values calculated below.

The length of a light pulse with duration $8 n s$ is $L_{P} \approx 2.4 \mathrm{~m}$. The amplified beam with the duration $\tau_{0} \approx 8 \mathrm{~ns}$, the divergence $\theta_{L} \approx 3 \times 10^{-4} \mathrm{rad}$, and the radius $R_{L} \approx 0.5 \mathrm{~cm}$ (Table 3) was focused by lens 9 . In accordance with Eq. (5), the length $L$ of the focal caustic varied from $L_{M I N} \approx 0.6 \mathrm{~mm}$ for $F=11 \mathrm{~cm}$ to $L_{M I D} \approx 1.5 \mathrm{~cm}$ for $F=50 \mathrm{~cm}$ and $L_{M A X} \approx 6 \mathrm{~cm}$ for $F=100 \mathrm{~cm}$. The long-pulse condition $L_{P} \gg L$ is met for the entire range of the values of $F$. At any moment of time, pump power $W_{L}$ can be treated as constant over the caustic length $L$, which is approximately equal to the length of the area where the nonlinear interaction occurs. To simplify the analysis, we assume that the pump intensity $I_{L}$ is uniformly distributed over a cylindrical focal caustic of diameter $d_{f}$ and length $L$.

It should be noted that such a technique is widely met in nonlinear optical studies. For instance, the impact of the focal caustic dimensions upon the optical breakdown threshold has been studied in [24]. Radiation of the ruby laser was focused by the lenses with focal lengths $F=1 \sim 13 \mathrm{~cm}$ into gases. The focal caustics were supposed to have cylindrical shapes, but their dimensions were not measured. The transition from the focal length to the focal spot diameter was performed with the help of equation $d_{f} \approx F \theta_{L}$, where $\theta_{L}$ is the laser beam divergence. In contrast to [24], long-focal-length lenses with $F \geq 11 \mathrm{~cm}$ were used under our experimental conditions. Moreover, due to the fluorescence of hexane the focal caustics were seen with the naked eye, and for $F=50$ and $100 \mathrm{~cm}$ their lengths could be measured by a ruler.

When a laser beam with the power $W_{L}$, the radius $R_{L}$, and the divergence $\theta_{L}$ is focused by a lens, the product $\left(I_{L} L\right)$ is independent of the focal length $F$ of this lens. Indeed,

$$
\begin{aligned}
& I_{L} \approx \frac{W_{L}}{S_{f}} \\
& S_{f}=\frac{\pi d_{f}^{2}}{4} \approx \frac{\pi\left(F \theta_{L}\right)^{2}}{4} \\
& L \approx \frac{d_{f}}{\theta_{0} / 2} \approx \frac{F \theta_{L}}{R_{L} / F}=\frac{F^{2} \theta_{L}}{R_{L}}
\end{aligned}
$$

where $W_{L}, R_{L}, \theta_{L}$ are independent of $F ; d_{f} \approx F \theta_{L} ; S_{f}$ is the cross-sectional area of the focal caustic; $\theta_{0} \approx 2 R_{L} / F$ is the convergence angle of the beam after the lens. We obtain

$$
I_{L} \propto F^{-2}, L \propto F^{2} \Rightarrow\left(I_{L} L\right) \approx \text { const }
$$

The full expression is 


$$
\left(I_{L} L\right) \approx \frac{4 W_{L}}{\pi \theta_{L} R_{L}} \approx \frac{4 U_{L}}{\pi \theta_{L} R_{L} \tau_{0}},
$$

where $U_{L}$ and $\tau_{0}$ are the energy of the pulse and its duration, respectively. For the parameters of the amplified beam presented in Table 3, Eq. (7) yields $\left(I_{L} L\right) \approx 3 \times 10^{3} \mathrm{MW} / \mathrm{cm}$. The amplified beam energy $3 \mathrm{~mJ}$ (Table 3 ) corresponds to the absence of diagnostic equipment and reflects the maximum possible value for this laser system before cell 10. Due to the diagnostical and other losses of energy between the output of amplifier 2 and the focal caustic of lens 9 , the real on-target energy is $U_{L} \approx 1.5 \mathrm{~mJ}$. Accordingly, we have $\left(I_{L} L\right) \approx 1.5 \times 10^{3} \mathrm{MW} / \mathrm{cm}$.

There are two varying parameters in our experiments - $\alpha$ and $F$. As far as the variation of $F$ is concerned, Eq. (6) shows that the value

$$
\left(I_{L} L\right) \approx 1.5 \times 10^{3} \mathrm{MW} / \mathrm{cm}
$$

is constant. Since $\left(I_{L} L\right) \approx$ const , the only parameter that may affect the left part of Eq. (2) via $G$ is $\alpha$. If the SS process does not involve the heating due to the linear absorption, $G$ is independent on $\alpha$, and the total gain $\left(I_{L} G L\right)$ does not change in our experiments.

The diameter of the amplified beam incident on lens 9 is $D_{L} \approx 1 \mathrm{~cm}$ (Table 3). The focal length of lens 9 is $F>10 \mathrm{~cm}$. Therefore, $D_{L} / F<0.1$, and the focal caustics were not distorted by spherical aberrations of lens 9 or by a jump in the refractive index across the air-cell boundary.

\subsection{The Parameters of SBS and Linear STS-2 for Our Experimental Conditions}

Since SBS and linear STS-2 were considered responsible for the observed SS in the previous studies (see Table 1), we should calculate their parameters for our experimental conditions.

\subsubsection{Properties of SBS}

According to the SBS model [1 - 5], the modulated electrostrictive force amplifies a hypersonic wave with the wave vector $\mathrm{q}=\mathrm{k}_{L}-\mathrm{k}_{S}$ and the frequency

$$
\Omega_{B}=|\mathrm{q}| v,
$$

where $\omega_{L}, \mathrm{k}_{L}$ and $\omega_{S}, \mathrm{k}_{S}$ denote the frequencies and the wave vectors of the pump and scattered waves; $\mathrm{V}$ is the speed of sound. The steady-state gain factor near the Stokes resonance peak is

$$
G_{B}=\frac{\rho^{2}(\partial \mathcal{E} / \partial \rho)^{2}}{1+\left(\omega_{L}-\omega_{S}-\Omega_{B}\right)^{2} / \Gamma_{B}^{2}} \cdot \frac{\omega_{S} \mathrm{q}^{2}}{4 \Omega_{B} \Gamma_{B} \rho n^{2} c^{2}},
$$

where $\rho$ is the density, $c$ is the speed of light, $n$ is the unperturbed refractive index. The decay rate $\Gamma_{B}$ is related to the decay time $\tau_{B}$ :

$$
\Gamma_{B}(\mathrm{q})=A|\mathrm{q}|^{2}, \tau_{B} \approx \Gamma_{B}^{-1} .
$$

For liquids, $A=\frac{2 \eta_{1}}{3 \rho}$, where $\eta_{1}$ is the shear viscosity. The highest gain factor (10) is achieved when $\omega_{L}-\omega_{S}=\Omega_{B}$. When the scattered wave propagates at an angle $\theta$ relative to the pump wave,

$$
q=|\mathrm{q}|=\left|\mathrm{k}_{L}-\mathrm{k}_{S}\right| \approx 2\left|\mathrm{k}_{L}\right| \sin (\theta / 2)=2\left(\omega_{L} n / c\right) \sin (\theta / 2) .
$$

Accordingly, the SBS frequency shift is

$$
\Omega_{B}=q v=2 \omega_{L} n(v / c) \sin (\theta / 2) .
$$

\subsubsection{Properties of Linear STS-2}

According to the linear STS-2 model $[1-4,13]$, the modulated heating amplifies a thermal wave with the wave vector $\mathrm{q}=\mathrm{k}_{L}-\mathrm{k}_{S}$. The steady-state gain factor is

$$
G_{T} \approx \frac{\omega_{S} \alpha}{\operatorname{cn} \rho c_{p} \Gamma_{T}}\left(\frac{\partial \varepsilon}{\partial T}\right) \frac{\left(\omega_{L}-\omega_{S}\right) / \Gamma_{T}}{1+\left(\omega_{L}-\omega_{S}\right)^{2} / \Gamma_{T}^{2}},
$$

where $\alpha$ is the linear absorption coefficient, $c_{p}$ is the specific heat, and $\chi=$ is the thermal diffusivity. The decay rate $\Gamma_{T}$ is related to the decay time $\tau_{T}$ :

$$
\Gamma_{T}=\chi|\mathrm{q}|^{2}, \tau_{T} \approx \Gamma_{T}^{-1} .
$$

Since $\frac{\partial \varepsilon}{\partial T}$ is negative for the most substances, a positive gain corresponds to the anti-Stokes frequency shift $\left(\omega_{L}-\omega_{S}\right)<0$. The highest gain factor (14) is achieved when $\left(\omega_{L}-\omega_{S}\right)=\Omega_{T}=-\Gamma_{T}$.

\subsubsection{The Predicted Values of the SBS and Linear STS-2 Parameters for Our Experiments}

Under our experimental conditions, the following numerical values $[2,5,25]$ should be substituted into Eqs. (9)-(15):

$$
\begin{aligned}
& \lambda=308 \mathrm{~nm}, \theta=\pi, \rho \approx 0.66 \frac{g}{\mathrm{~cm}^{3}}, n \approx 1.4, \eta_{1} \approx 3.2 \times 10^{-3} P \approx 3.2 \times 10^{-3} \frac{\mathrm{g}}{\mathrm{cms}}, \\
& v \approx 10^{5} \frac{\mathrm{cm}}{\mathrm{s}}, c \approx 3 \times 10^{10} \frac{\mathrm{cm}}{\mathrm{s}}, \rho\left(\frac{\partial \varepsilon}{\partial \rho}\right)_{T} \approx 1, \frac{\omega_{S}}{2 \pi} \approx \frac{\omega_{L}}{2 \pi} \approx 10^{15} \mathrm{~Hz}, \chi \approx 10^{-3} \frac{\mathrm{cm}^{2}}{\mathrm{~s}}, \\
& \left(\frac{\partial \varepsilon}{\partial T}\right)_{P} \approx-1.5 \times 10^{-3} \mathrm{~K}^{-1}, c_{P} \approx 1.5 \frac{J}{\mathrm{~cm}^{3} \mathrm{~K}}, \delta=\frac{c_{P}}{c_{V}} \approx 1.3 .
\end{aligned}
$$

The predicted SBS frequency shift is $\Omega_{B}=0.33 \mathrm{~cm}^{-1}$ (see Table 2). The hypersonic grating decay time is $\tau_{B} \approx 1 \mathrm{~ns}$. Since this time is shorter than the pump duration by a factor of 8 , the steady-state model can be used. The frequency-optimized SBS gain factor (10) is expressed as 


$$
G_{B}=\frac{\omega_{S} \mathrm{q}^{2}}{4 \Omega_{B} \Gamma_{B} \rho n^{2} c^{2}}=\frac{3}{16 n^{3} c v \eta_{1}} \approx 0.07 \frac{\mathrm{cm}}{M W}
$$

The predicted linear STS-2 frequency shift is $\Omega_{T} \approx-\Gamma_{T} \approx-0.01 \mathrm{~cm}^{-1}$. The thermal grating decay time is $\tau_{T} \approx 3 n s$. Even though it is shorter than the pump duration by almost a factor of 3 , we can not reliably use the steady-state model. Since we explore the possibility of the emergence of linear STS-2 in an experiment rather than attempt to maximize its efficiency, we do use the steady-state model to simplify the further analysis. The thermal-grating wavelength $\Lambda_{T}$ associated with the backscattering is

$$
\Lambda_{T}=\frac{2 \pi}{q} \approx \frac{2 \pi}{2 k_{L}}=\frac{\lambda}{2 n} \approx 10^{-7} \mathrm{~m} .
$$

The time of the pressure relaxation over $\Lambda_{T}$ is

$$
\tau_{p r}=\frac{\Lambda_{T}}{v} \approx 10^{-10} \mathrm{~s}
$$

Since $\tau_{p r}<<\tau_{0}, G_{T}$ can be obtained by using the permittivity temperature derivative at constant pressure. The frequency-optimized linear STS-2 gain factor (14) is expressed as

$$
G_{T}=\frac{\omega_{S} \alpha}{2 \operatorname{cn} \rho c_{P} \Gamma_{T}}\left(\frac{\partial \varepsilon}{\partial T}\right)_{P}=\frac{\alpha c}{8 \omega_{L} n^{3} \rho c_{P} \chi}\left(\frac{\partial \varepsilon}{\partial T}\right)_{P} \approx 0.2 \alpha \frac{\mathrm{cm}^{2}}{M W}
$$

The frequency-optimized gain factors and total gains for linear STS-2 and SBS calculated for various values of $\alpha$, but for constant $\left(I_{L} L\right) \approx 1.5 \times 10^{3} \mathrm{MW} / \mathrm{cm}$, as in our experiments, are listed in Table 4 . The first column of the table shows the linear absorption coefficient $\alpha$; the second and fourth columns - the gain factors $G_{T}$ and $G_{B}$; the third and the last columns - the total gains $\left(I_{L} G_{T} L\right)$ and $\left(I_{L} G_{B} L\right)$.

Table 4. Gain factors $G_{T}, G_{B}$ and total gains $\left(I_{L} G_{T} L\right),\left(I_{L} G_{B} L\right)$ for linear STS-2 and SBS calculated for various values of $\alpha$, but for constant $\left(I_{L} L\right) \approx 1.5 \times 10^{3} \mathrm{MW} / \mathrm{cm}$ (as in our experiments).

\begin{tabular}{lllll}
\hline \multirow{2}{*}{$\alpha, \mathrm{cm}^{-1}$} & Linear STS-2 & \multicolumn{3}{c}{ SBS } \\
\cline { 2 - 5 } & $G_{T}, \mathrm{~cm} / \mathrm{MW}$ & $\left(I_{L} G_{T} L\right)$ & $G_{B}, \mathrm{~cm} / \mathrm{MW}$ & $\left(I_{L} G_{B} L\right)$ \\
\hline 0.01 & 0.002 & 3 & 0.07 & 105 \\
0.046 & 0.009 & 14 & 0.07 & 105 \\
0.1 & 0.02 & 30 & 0.07 & 105 \\
\hline
\end{tabular}

The theoretical estimates presented in Table 4 lead to the following conclusions.

Linear STS-2. The total gain (depending on $\alpha$ via $G_{T}$ ) is much lower than the threshold given by Eq. (2) both for the "chemically pure" ( $\alpha=0.046 \mathrm{~cm}^{-1}$ ), and a fortiori for the chromatography-grade $\left(\alpha=0.01 \mathrm{~cm}^{-1}\right)$ hexane. The threshold is reached when $\alpha=\alpha_{S T S}^{T H R} \approx 0.1 \mathrm{~cm}^{-1}$.
SBS. The total gain (independent of $\alpha$ ) is higher than the threshold given by Eq. (2).

\subsection{The Emergence and Disappearance of the Spectral Components in Accordance with the Existing Theory}

\subsubsection{The Shifted Component of the Stimulated Backscatter Spectrum}

Since the total gain $\left(I_{L} G_{B} L\right)$ (Table 4) exceeds the threshold (2) and the measured value of the frequency shift $0.33 \mathrm{~cm}^{-1}$ (Figs. 2b and 2c) agrees with Eq. (13) (Table 2), the shifted component should be attributed to SBS.

To explain the absence of the shifted component in Fig. 2a, we explore the possibility of the SBS breakdown. The only mechanism that can suppress SBS under our conditions is the phase mismatch $[11,12]$. When the permittivity of the medium changes with time due to the weak uniform heating caused by the linear absorption with the coefficient $\alpha$, the wavelengths of the pump and scattered waves vary. Since the acoustic wave has a finite response time, the phase matching conditions are violated. If $I_{L}(L)$ is the input pump intensity and $I_{S}(L)$ is the output backscattered intensity, then the SBS conversion efficiency over the length $L$ of nonlinear interaction is $\eta=\frac{I_{S}(L)}{I_{L}(L)}$. The dependence of $\eta$ on $I_{L}(L)$ is given by the relation $[11,12]$ :

$$
h(D+\ln \eta)=\Gamma_{B} G_{B} \operatorname{arctg}\left\{I_{L}(L)(1-\eta) L h / \Gamma_{B}\right\}
$$

where $D \approx 30, G_{B}$ is the peak backward SBS gain factor defined by Eq. (16), and

$$
h=\frac{\alpha \omega_{L}}{2 \operatorname{cn} \rho c_{p}}\left(\frac{\partial \varepsilon}{\partial T}\right)_{P} .
$$

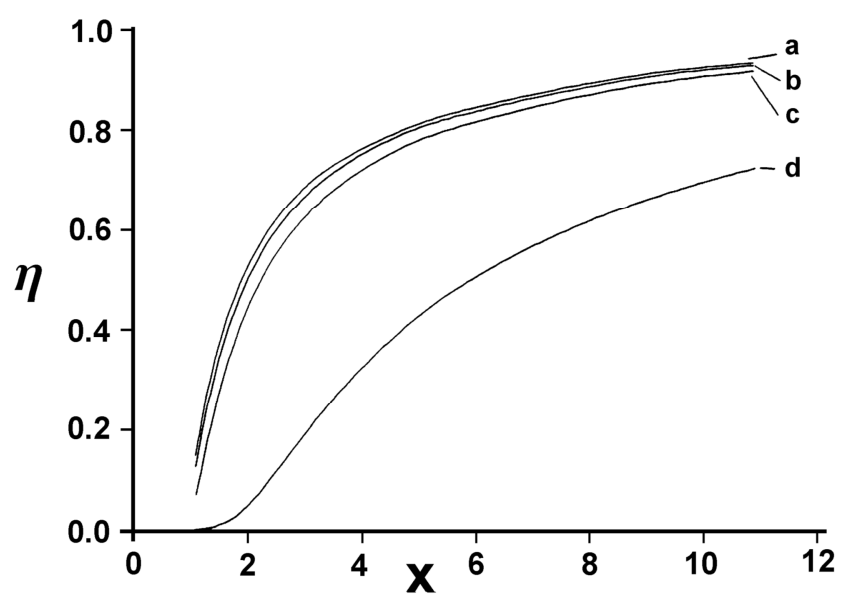

Fig. 4. Predicted SBS conversion efficiency $\eta$ vs. the total gain normalized by its threshold value $x=\frac{\left(I_{L} G_{B} L\right)}{D}$ for hexane, for $\lambda=308 \mathrm{~nm}$ and $\alpha=(a) 0.0001,(b) 0.03,(c) 0.05,(d) 0.1 \mathrm{~cm}^{-1}$. 
Figure 4 shows the SBS conversion efficiency $\eta$ as a function of the total gain normalized by its threshold value $x=\frac{\left(I_{L} G_{B} L\right)}{D}$, evaluated numerically by using the relation $(20)$ for hexane, for $\lambda=308 \mathrm{~nm}$, and for several values of $\alpha$. The critical value corresponding to curve " $\mathrm{d}$ " is $\alpha_{C R} \approx 0.1 \mathrm{~cm}^{-1}$. For $\alpha=0.01,0.046,0.08 \mathrm{~cm}^{-1}$, no SBS suppression due to phase mismatch should be expected.

\subsubsection{The Unshifted Component of the Stimulated Backscatter Spectrum}

The PC via SS can be caused by mechanisms other than SBS $[1,3,4]$. Since the measured frequency shift does not exceed $0.02 \mathrm{~cm}^{-1}$ (the experimental error), we should consider the stimulated thermal scattering caused by heating due to the electrocaloric effect (STS-1), the linear STS-2 and the stimulated Raleigh wing scattering (SRWS).

First, consider the SRWS. Since the molecular anisotropy of liquid hexane is weak [26], the excitation of SRWS is impossible. Normally, the SRWS gain factor for liquids does not exceed $G_{R W} \approx 10^{-3} \mathrm{~cm} / M W$ [1]. For our condition (8) the SRWS total gain does not exceed $\left(I_{L} G_{R W} L\right) \approx 1.5$, which is much lower than the threshold (2).

Now consider the STS-1. According to [1], the STS-1 to SBS gain factor ratio is

$$
\frac{G_{R L}}{G_{B}}=\frac{(\delta-1) c \Gamma_{B}}{4 \pi v \omega_{S}}
$$

For our experimental conditions, $\frac{G_{R L}}{G_{B}} \leq 10^{-2}$. From the value of $\left(I_{L} G_{B} L\right)$ in the last column of Table 4, the STS-1 total gain is $\left(I_{L} G_{R L} L\right) \leq 1$, which is much lower than the threshold (2).

The processes of SRWS and STS-1 do not involve heating due to absorption of the radiation, and their total gains do not change in our experiments as long as $\left(I_{L} L\right)$ is constant (see Section 6.2). The emergence of SRWS and STS-1 components under our experimental conditions is impossible.

Now consider linear STS-2. The predicted frequency shift $\Omega_{T} \approx-0.01 \mathrm{~cm}^{-1}$ (see Section 6.3.3) agrees with the measured one. Indeed, the absolute value of the latter is less than $0.02 \mathrm{~cm}^{-1}$ (the experimental error). Heating due to absorption of the radiation is involved in the linear STS-2 process, and its total gain varies with $\alpha$ while $\left(I_{L} L\right)$ is constant. The emergence of linear STS-2 component under our experimental conditions (Table 4) is possible only when $\alpha \geq 0.1 \mathrm{~cm}^{-1}$.

\subsection{Decrease in Phase-Conjugation Fidelity in Accordance with the Existing Theory}

The only mechanism that can cause a decrease in the PC fidelity (Figs. 3b and 3c) under our experimental conditions is the phase self-modulation $[11,12]$. Consider the following cylindrically symmetric problem in coordinates $(R, z, t)$. A wave with a plane front and a spatially nonuniform intensity $I_{L}(R, t)$ enters a nonlinear medium at $t=0$ across the boundary located at $z=0$ and propagates towards the boundary located at $z=L$. The medium is characterized by the linear absorption coefficient $\alpha$. Heat conduction does not contribute in transverse coordinate $R$. The temperature increment over time $t$ is

$$
\delta T(R, t)=\frac{1}{\rho c_{P}} \int_{0}^{t} \alpha I_{L}(R, \tau) d \tau=\frac{\alpha}{\rho c_{P}} \int_{0}^{t} I_{L}(R, \tau) d \tau
$$

The refractive index depends on temperature: $n=n_{0}+\delta n(T)$, where the nonlinear term is

$$
\delta n(R, t)=\left(\frac{\partial n}{\partial T}\right) \delta T(R, t)=\left(\frac{\partial n}{\partial T}\right) \frac{\alpha}{\rho c_{P}} \int_{0}^{t} I_{L}(R, \tau) d \tau
$$

The wave vector is $\mathrm{k}(R, t) \approx \mathrm{e}_{z} k(R, t)$, where $k=k_{0}+\delta k=\frac{\omega}{c}\left(n_{0}+\delta n\right)$.

The nonlinear phase increment over distance $L$ is

$$
\begin{aligned}
& \delta \Phi(R, t)=\delta k L=\frac{\omega L}{c} \delta n(R, t)=\frac{\omega L}{c}\left(\frac{\partial n}{\partial T}\right) \frac{\alpha}{\rho c_{P}} \int_{0}^{t} I_{L}(R, \tau) d \tau= \\
& =\frac{\omega}{c}\left(\frac{\partial n}{\partial T}\right) \frac{\alpha}{\rho c_{P}} \int_{0}^{t} I_{L}(R, \tau, F) L(F) d \tau .
\end{aligned}
$$

A wave with a plane front at $z=0$ will have a radially nonuniform front at $z=L$. The front distortion is zero at $t=0$ and maximal at the end of the pulse.

Due to the phase self-modulation of the pump wave the backscattered wave gets coupled to the dynamically distorted beam. Taking into account the finite nonlinearity decay time, this effect may cause a decrease in PC fidelity.

It must be noted that lenses 9 as well as cells 10 (see Fig. 1) were changed only in the intervals between separate sets of experiments. The SS of a great number of pump pulses was studied in every set. That is why $F$ is a parameter in the final form of expression (24), which remains invariant over the pump duration. For pump pulses from different sets of experiments, at any instant $\tau$ the product $\left(I_{L} L\right)$ contained in (24) does not depend on $F$, as shown by Eq. (6). Therefore, $\delta \Phi(R, t)$ does not depend on $F$, and the heating with $\alpha=$ const can not explain the decrease in the PC fidelity when $F$ is reduced from 100 to $11 \mathrm{~cm}$.

\subsection{The Implication of Two-Photon Absorption in the Theory of Stimulated Scattering}

The existing SS theory developed for linearly absorbing media cannot explain our experimental results. First, since the linear absorption with $\alpha<0.1 \mathrm{~cm}^{-1}$ is not sufficiently strong to give rise to linear STS-2, the physical mechanism 
responsible for the unshifted component observed for $\alpha=0.01 \sim 0.08 \mathrm{~cm}^{-1}$ (Figs. 2a and 2b) remains unclear. Secondly, since the linear absorption with $\alpha<0.1 \mathrm{~cm}^{-1}$ is not strong enough to suppress SBS via the phase mismatch, the disappearance of the SBS component when $F$ is reduced from 50 to $11 \mathrm{~cm}$ (Figs. $2 \mathrm{a}$ and $2 \mathrm{~b}$ ) observed for $\alpha=0.01 \sim 0.08 \mathrm{~cm}^{-1}$ is yet to be explained. Thirdly, the linear absorption with $\alpha=0.01 \mathrm{~cm}^{-1}=$ const cannot explain the substantial decrease in the PC fidelity caused by the decrease in $F$ from 100 to $11 \mathrm{~cm}$ (Figs. $3 \mathrm{~b}$ and $3 \mathrm{c}$ ).

The two-photon absorption should be expected in hexane for $\lambda<400 \mathrm{~nm}$ [27]. Suppose that our experiments were strongly affected by the two-photon absorption. More specifically, assume that the unshifted component corresponds to the STS-2 process associated with the heating caused by the two-photon absorption. We call this new mechanism the two-photon STS-2, as distinct from the previously known mechanism induced by the linear (single-photon) absorption, i.e., linear STS-2.

The intensity $I(z)$ of the radiation propagating along $z$ axis is governed by the following equation $[1,28]$

$$
\frac{d I}{d z}=-\gamma I^{2}
$$

where $\gamma$ is the two-photon absorption coefficient. Its solution is

$$
I(z)=\frac{I_{0}}{1+I_{0} \gamma z},
$$

where $I_{0}=I(0)$. For the weak two-photon absorption $\left(I_{0} \gamma z<<1\right)$, this solution gets reduced to

$$
I(z)=I_{0}\left(1-I_{0} \gamma z\right)
$$

In our experiments, we have the condition (8) and $\gamma \approx 10^{-4} \mathrm{~cm} / M W$ (see Section 6.7). Thus, $\left(I_{L} \gamma L\right) \approx 0.15$ and Eq. (27) can be used.

The weak linear absorption with coefficient $\alpha$ is described by the equation

$$
I(z)=I_{0}(1-\alpha z) .
$$

It follows from Eqs. (27) and (28) that in our experiments $\left(I_{L} \gamma\right)$ plays the role of $\alpha$. We can define the total absorption coefficient as

$$
\alpha_{\Sigma}=\alpha+\left(I_{L} \gamma\right)
$$

where $\left(I_{L} \gamma\right)$ is the two-photon contribution. The equivalence should be understood in a quantitative sense. The linear (single-photon) and the two-photon absorption can be treated as similar processes only with respect to the final result of the resonant light-matter interaction, i.e., the irreversible dissipation of the electromagnetic energy into the thermal one. Linear and two-photon absorption are different in terms of quantum mechanical schemes and expressions for the absorption cross-section.

Physical mechanisms of linear STS-2 and two-photon STS-2 are essentially different, being roughly similar with respect to transformation of temperature fluctuations into permittivity ones.

Why $I_{0}$ is replaced by $I_{L}$ in Eqs. (27)-(29)? Due to a complexity of the theoretical task including several connected linear and nonlinear effects we made an approximation that our pump intensity does not change along the nonlinear interaction length. In accordance with this approximation we replaced the input intensity $I_{0}$ with the pump intensity $I_{L}$ in Eqs. (27)-(29). This approximation is based on the following consideration. The amplified beam parameters provided conditions for SS very close to its threshold, and the strong pump saturation does not take place. As it follows from the title of this article there are two main objectives of this study: first, to obtain a pure two-photon STS-2 spectral line, and second, to obtain a pure SBS spectral line. The two-photon STS-2 mechanism should dominate for as hard as possible focusing with as weak as possible linear absorption, i.e. for $F=11 \mathrm{~cm}$ and $\alpha_{M I N}=0.01 \mathrm{~cm}^{-1}$. The caustic length is $L_{M I N}=0.06 \mathrm{~cm}$. Even for $\alpha_{M A X}=0.17 \mathrm{~cm}^{-1}$, $\alpha_{M A X} L_{M I N}=0.01$, and $\exp (-0.01)=0.99$. The linear absorption is negligible. The SBS mechanism should dominate for as soft as possible focusing with as weak as possible linear absorption, i.e. for $F=100 \mathrm{~cm}$ and $\alpha_{M I N}=0.01 \mathrm{~cm}^{-1}$. The caustic length is $L_{M A X}=6 \mathrm{~cm}$, $\alpha_{M I N} L_{M A X}=0.06$, and $\exp (-0.06)=0.94$. The linear absorption is also negligible.

The observation of the "linear STS-2" in the previous study [22] is not clear (see Section 3.3). Therefore, linear STS-2 also calls for further investigation. For $\alpha=0.046 \mathrm{~cm}^{-1}$, $\alpha L_{\text {MAX }}=0.27$ and $\exp (-0.27)=0.76$. The linear absorption is weak. For $\alpha_{S T S}^{T H R} \approx 0.1 \mathrm{~cm}^{-1}, \alpha_{S T S}^{T H R} L_{M A X}=0.6$ and $\exp (-0.6)=0.55$. Under our experimental conditions the linear absorption becomes considerable only for $F=100 \mathrm{~cm}$ (i.e., $L=L_{M A X}$ ) and $\alpha \geq \alpha_{S T S}^{T H R} \approx 0.1 \mathrm{~cm}^{-1}$.

The two-photon absorption under our experimental conditions is constant and equal to $\left(I_{L} \gamma L\right) \approx 0.15$ (see Section 6.6), and $\exp (-0.15)=0.86$. The two-photon absorption is weak.

\subsection{The Calculation of Two-Photon Absorption Cross-Section from the Threshold Pump Intensity for Two-Photon STS-2}

When $\left(I_{L} L\right)=$ const, the linear STS-2 occurs if $\alpha \geq \alpha_{S T S}^{T H R}$. The two-photon STS-2 must also exhibit threshold behavior, 
but with respect to $\left(I_{L} \gamma\right)$ rather than $\alpha$. When material properties $\alpha$ and $\gamma$ are kept constant, an increase in $I_{L}$ caused by a decrease in $F$ leads to different consequences for the total gain $\left(I_{L} G_{T} L\right)$. It remains invariant for the linear STS-2 and increases for the two-photon STS-2, because $G_{T} \propto \alpha=$ const (see Eq. (14)) in the former case and $G_{T} \propto\left(I_{L} \gamma\right) \propto I_{L}$ in the latter.

The linear STS-2 and the two-photon STS-2 can be distinguished experimentally. If the medium with $\alpha$ and $\gamma$ such that $\alpha,\left(I_{L} \gamma\right) \ll<\alpha_{S T S}^{T H R}$ is used and $I_{L}$ is gradually increased while $\left(I_{L} L\right)$ is kept constant, the pure two-photon STS-2 component must appear in the spectrum when $\left(I_{L} \gamma\right) \approx \alpha_{S T S}^{T H R}$.

Let us find $\left(I_{L} \gamma\right)$. The total gain for STS- 2 caused both by the linear and the two-photon absorption (29) reaches the threshold (2) when $\alpha_{\Sigma}^{T H R} \approx \alpha_{S T S}^{T H R} \approx 0.1 \mathrm{~cm}^{-1}$ (Table 4). For $\alpha=0.01 \mathrm{~cm}^{-1}$, the unshifted component appears in the backscatter spectrum when $I_{L}^{T H R} \approx 10^{9} \mathrm{~W} / \mathrm{cm}^{2}(F=50 \mathrm{~cm})$ (Fig. 2b). When $I_{L} \approx 2.5 \times 10^{8} \mathrm{~W} / \mathrm{cm}^{2}(F=100 \mathrm{~cm})$ no unshifted component (Fig. 2c), i.e., the threshold is not reached. Since $\alpha=0.01 \mathrm{~cm}^{-1}<<\alpha_{S T S}^{T H R} \approx 0.1 \mathrm{~cm}^{-1}$, the unshifted component must be attributed to the pure two-photon STS-2 mechanism. The threshold pump intensity $I_{L}^{T H R} \approx 10^{9} \mathrm{~W} / \mathrm{cm}^{2}$ can be used to obtain the estimate

$$
\alpha_{\Sigma}^{T H R}=\alpha+\left(I_{L}^{T H R} \gamma\right) \approx\left(I_{L}^{T H R} \gamma\right) \approx \alpha_{S T S}^{T H R} \approx 0.1 \mathrm{~cm}^{-1}
$$

For a particular pump wavelength (for $\lambda=308 \mathrm{~nm}$ in our case) coefficient $\gamma$ describes the property of the medium (of the chromatography-grade hexane in our case). If $\left(I_{L} \gamma\right)$ is known for one value of $I_{L}$ (for $I_{L}^{T H R}$ in our case), then it can readily be calculated for other values of $I_{L}$. The first column of Table 5 gives the three focal lengths $F$ of lens 9 used in our experiments; the second column - the three corresponding pump intensities $I_{L}$; and the last column - the two-photon contribution $\left(I_{L} \gamma\right)$ to the total absorption coefficient (29).

Table 5. The two-photon contribution $\left(I_{L} \gamma\right)$ to the total absorption coefficient (29) of hexane at $\lambda=308 \mathrm{~nm}$ for pump intensities $I_{L}$ corresponding to the three focal lengths $F$ of lens 9 used in our experiments.

\begin{tabular}{lll}
\hline$F, \mathrm{~cm}$ & $I_{L}, W / \mathrm{cm}^{2}$ & $\left(I_{L} \gamma\right), \mathrm{cm}^{-1}$ \\
\hline 11 & $\geq 10^{10}$ & $\geq 1.0$ \\
50 & $10^{9}$ & $\approx 0.1$ (experiment) \\
100 & $2.5 \times 10^{8}$ & $\approx 0.025$ \\
\hline
\end{tabular}

From $\left(I_{L}^{T H R} \gamma\right) \approx 0.1 \mathrm{~cm}^{-1}$ and $I_{L}^{T H R} \approx 10^{9} \mathrm{~W} / \mathrm{cm}^{2}$, we can calculate the two-photon absorption coefficient $\gamma$ and the cross-section $\sigma_{2}$. Indeed,

$$
\begin{gathered}
\gamma=\frac{I_{L}^{T H R} \gamma}{I_{L}^{T H R}} \approx \frac{0.1\left[\mathrm{~cm}^{-1}\right]}{10^{9}\left[W / \mathrm{cm}^{2}\right]} \approx 10^{-10}[\mathrm{~cm} / W] \approx 10^{-4}[\mathrm{~cm} / \mathrm{MW}] \\
\left(I_{L} \gamma\right)=\sigma_{2} N \frac{I_{L}}{\hbar \omega},
\end{gathered}
$$

where $\frac{I_{L}}{\hbar \omega}$ is the pump intensity in the units of [ photon $\times \mathrm{cm}^{-2} \times \mathrm{s}^{-1}$ ], $N=\frac{\rho}{M}$ is the molecular density $(M$ is the molecular mass). For hexane $\left(\mathrm{C}_{6} \mathrm{H}_{14}\right) \quad N \approx 4 \times 10^{21} \mathrm{~cm}^{-3}$. The photon energy corresponding to $\lambda=308 \mathrm{~nm}$ is $\hbar \omega \approx 4 \mathrm{eV} \approx 6.4 \times 10^{-19} \mathrm{~J}$. We have

$$
\sigma_{2}=\frac{\hbar \omega\left(I_{L}^{T H R} \gamma\right)}{I_{L}^{T H R} N} \approx(2 \pm 1) \times 10^{-50} \mathrm{~cm}^{4} s
$$

The accuracy is estimated as

$$
\frac{\delta \sigma_{2}}{\sigma_{2}} \approx \frac{\delta \alpha^{T H R}}{\alpha^{T H R}}+\frac{\delta I_{L}}{I_{L}} \approx 0.2+0.3 \approx 0.5
$$

where $\alpha^{T H R}$ is the experimental threshold value for the linear STS-2.

For comparison, consider the two-photon nonresonant ionization of a many-electron atom at a moderate electric field strength, which does not involve the near-threshold absorption, the multiple ionization, and the perturbation of the atomic spectra. A typical cross-section of this process is $\sigma_{2}^{i} \approx 10^{-49} \sim 10^{-48} \mathrm{~cm}^{4} s[29,30]$. Normally, $\sigma_{2}^{i}$ is smaller than the cross-section for the two-photon bound-bound transition at the absorption-line center. The value (33) extracted from the experimental data is smaller than $\sigma_{2}^{i}$. This substantiates our assumption that the main contribution to the STS-2 gain is made by the two-photon absorption. The relatively small value of $\sigma_{2}$ may be explained by the fact that the two-photon absorption scheme $(\lambda / 2=154 \mathrm{~nm}$, hexane) corresponds to a wing of the absorption line.

\subsection{Phase Mismatch for SBS Caused by Two-Photon Heating}

The relationship between $I_{L},\left(I_{L} \gamma\right)$ and $F$ (see Table 5) explains why the SBS component is not observed for $\alpha=0.01 \mathrm{~cm}^{-1}$ when $F=11 \mathrm{~cm}$ (Fig. 2a). Indeed, in this case $I_{L} \geq 10^{10} \mathrm{~W} / \mathrm{cm}^{2}$, and the total absorption coefficient $\alpha_{\Sigma}=\alpha+\left(I_{L} \gamma\right) \approx\left(I_{L} \gamma\right) \geq 1.0 \mathrm{~cm}^{-1}$ is much larger than the critical value $\alpha_{C R} \approx 0.1 \mathrm{~cm}^{-1}$ corresponding to the phase mismatch (Fig. 4). The heating due to the two-photon absorption is sufficiently strong to cause an SBS breakdown. 


\subsection{The Phase Self-Modulation and the Decrease in Phase-Conjugation Fidelity Caused by Two-Photon Heating}

We consider only the case of Fig. 3 ( $\alpha=0.01 \mathrm{~cm}^{-1}=$ const $)$, when the linear absorption (Table 5) and the linear STS-2 (Table 4) do not contribute. The case of the heating due to the linear absorption was discussed in [11, 12].

Assuming that the two-photon absorption plays the dominant role, we replace $\alpha$ with $\left(I_{L} \gamma\right)$ in Eqs. (22)-(24), and the nonlinear phase increment over distance $L$ becomes

$$
\delta \Phi(R, t, F)=\delta k L=\frac{\omega}{c}\left(\frac{\partial n}{\partial T}\right) \frac{\gamma}{\rho c_{P}} \int_{0}^{t} I_{L}^{2}(R, \tau, F) L(F) d \tau
$$

The quantity $\left(I_{L}^{2} L\right)=I_{L}\left(I_{L} L\right)$ in Eq. (35) increases with $I_{L}$ as $\left(I_{L} L\right)=$ const, i.e. $\delta \Phi(R, t, F)$ depends on $F$. The two-photon heating explains the increase in the phase self-modulation of the pump wave when $F$ is reduced from 100 to $11 \mathrm{~cm}$.

The hypersonic grating decay time $\tau_{B} \approx 1 n s$ (see Section 6.3.3) is shorter than the pump duration by a factor of 8 . The phase hologram adjusts to the dynamic variations of the pump field caused by the phase self-modulation due to the two-photon heating. The SBS ensures a high PC fidelity.

The thermal grating decay time $\tau_{T} \approx 3 n s$ (see Section 6.3.3) is shorter than the pump duration by a factor of 3 . The phase hologram cannot keep pace with the dynamic variations of the pump field caused by the phase self-modulation due to the two-photon heating. Since the pump wave is scattered by a retarded hologram, the PC fidelity for the two-photon STS-2 loses quality.

Table 6. Physical mechanisms of the stimulated backscattering of $\mathrm{XeCl}$ $(\lambda=308 \mathrm{~nm})$ laser pulses with the duration $\tau_{0} \approx 8 \mathrm{~ns}$ in hexane characterized by two typical values of the linear absorption coefficient $\alpha$, for pump intensities $I_{L}$ corresponding to the three focal lengths $F$ of lens 9 , and $\left(I_{L} L\right) \approx 1.5 \times 10^{3} \mathrm{MW} / \mathrm{cm}$ ( $L$ is the nonlinear interaction length).

\begin{tabular}{|c|c|c|c|}
\hline \multirow[t]{2}{*}{$F, \mathrm{~cm}$} & \multirow{2}{*}{$I_{L}, W / \mathrm{cm}^{2}$} & \multicolumn{2}{|c|}{$\begin{array}{l}\text { Physical mechanisms of stimulated } \\
\text { backscattering }\end{array}$} \\
\hline & & $\alpha=0.01 \sim 0.08 \mathrm{~cm}^{-1}$ & $\alpha=0.17 \mathrm{~cm}^{-1}$ \\
\hline 100 & $2.5 \times 10^{8}$ & SBS & Linear STS-2 \\
\hline 50 & $10^{9}$ & $\begin{array}{l}\text { SBS } \\
+ \\
\text { Two-photon STS-2 }\end{array}$ & $\begin{array}{l}\text { Linear STS-2 } \\
+ \\
\text { Two-photon }\end{array}$ \\
\hline 11 & $\geq 10^{10}$ & Two-photon STS-2 & STS-2 \\
\hline
\end{tabular}

\subsection{Stimulated Scattering Mechanisms Consistent with the Present Studies}

Table 6 summarizes our experimental and theoretical studies of stimulated backscattering of $\mathrm{XeCl}(\lambda=308 \mathrm{~nm})$ laser pulses with the duration $\tau_{0} \approx 8 n s$ in liquid hexane. The first two columns show the three focal lengths $F$ of lens 9 used in our experiments and the corresponding pump intensities $I_{L}$. The last two columns list the revealed physical mechanisms of the stimulated backscattering in hexane characterized by two typical values of the linear absorption coefficient $\alpha=0.01 \sim 0.08 \mathrm{~cm}^{-1}$ and $\alpha=0.17 \mathrm{~cm}^{-1}$. Since the nonlinear region is the focal caustic of length $L$, the quantity $\left(I_{L} L\right) \approx 1.5 \times 10^{3} \mathrm{MW} / \mathrm{cm}$ remains unchanged for our experiments.

An analysis of the case $I_{L} \approx 2.5 \times 10^{8} \mathrm{~W} / \mathrm{cm}^{2}(F=100 \mathrm{~cm})$ where the two-photon absorption is insignificant (see Table 5), leads to the following conclusions.

1. The presence of linear STS-2 for $\alpha=0.17 \mathrm{~cm}^{-1}$ and its absence for $\alpha=0.01 \sim 0.08 \mathrm{~cm}^{-1}$ agree with the estimated threshold value $\alpha_{S T S}^{T H R} \approx 0.1 \mathrm{~cm}^{-1}$ (see Table 4).

2. The absence of SBS for $\alpha=0.17 \mathrm{~cm}^{-1}$ and its presence for $\alpha=0.01 \sim 0.08 \mathrm{~cm}^{-1}$ are consistent with the theoretical critical value for the phase mismatch $\alpha_{C R} \approx 0.1 \mathrm{~cm}^{-1}$ (see Fig. 4).

3. The absence of the SBS + linear STS-2 combination is explained by the fact that $\alpha_{S T S}^{T H R} \approx 0.1 \mathrm{~cm}^{-1}$ is close to $\alpha_{C R} \approx 0.1 \mathrm{~cm}^{-1}$, while the step of the variation of $\alpha$ from 0.08 to $0.17 \mathrm{~cm}^{-1}$ in our experiments is not sufficiently small to observe both these mechanisms simultaneously.

The presence of the SBS + two-photon STS-2 combination indicates that the step of the variation of $\alpha_{\Sigma}=\alpha+\left(I_{L} \gamma\right)$ in our experiments is sufficient for a simultaneous observation of both mechanisms.

The presence of the linear STS-2 + two-photon STS-2 combination is yet to be substantiated. These two mechanisms are virtually impossible to separate in our experimental spectra. Our theoretical analysis shows that neither linear STS-2 nor two-photon STS-2 can be singled out as the dominant mechanism when $\alpha \geq 0.1 \mathrm{~cm}^{-1}$ and $I_{L} \geq 10^{9} \mathrm{~W} / \mathrm{cm}^{2}$.

\subsection{Experimental Observation of a Genuine SBS Line in the Near-Ultraviolet Region}

According to the existing theory, which takes into account the linear absorption (see Table 4 and Fig. 4), only SBS can play a role in our experiments when $\alpha<0.1 \mathrm{~cm}^{-1}$, while only the linear STS-2 can contribute when $\alpha>0.1 \mathrm{~cm}^{-1}$ (in view of phase mismatch for SBS). It is not surprising that these two mechanisms are listed in the last column of Table 1 as those suggested in the previous studies.

Both "SBS" and "linear STS-2" in Table 1 are incorrect. Indeed, it follows from the third column of Table 1 that $I_{L} \geq 10^{10} \mathrm{~W} / \mathrm{cm}^{2}$ in all the previous studies. However, according to Table 6 , for $I_{L} \geq 10^{10} \mathrm{~W} / \mathrm{cm}^{2}$, either the two-photon STS-2 or the linear STS-2 + two-photon STS-2 combination should be observed in hexane when $\alpha<0.1 \mathrm{~cm}^{-1}$ and $\alpha>0.1 \mathrm{~cm}^{-1}$, respectively. Therefore "SBS" 
should be replaced with the two-photon STS-2 in all rows of Table 1 with the exception of the last one, while the "linear STS-2" should be replaced with the linear STS-2 + two-photon STS-2 combination in the last row. This consideration is corroborated by the fact that the measured frequency shift $\Omega$ in the fourth column of Table 1 is much less than the Brillouin shift $\Omega_{B}$ predicted theoretically for the same $\lambda$ (Table 2). It can be concluded that the genuine SBS line has never been observed in all previous studies of the stimulated scattering in the near-UV region.

In our experiments the stimulated scattering of the near-UV $(\lambda=308 \mathrm{~nm}$ ) laser radiation in hexane was studied, and the SBS line with the frequency shift equal to $0.33 \mathrm{~cm}^{-1}$ (see Figs. $2 \mathrm{~b}$ and $2 \mathrm{c}$ ) in full agreement with the theory (see Table 2) was observed. Thus, we were the first to observe the genuine SBS line in the near-UV region.

To grasp the genuine SBS line, we had to reduce the pump intensity from $I_{L} \geq 10^{10} \mathrm{~W} / \mathrm{cm}^{2}$ (Fig. 2a) as in the previous studies (Table 1) to $I_{L} \leq 10^{9} \mathrm{~W} / \mathrm{cm}^{2}$ (Figs. $2 \mathrm{~b}$ and 2c), while keeping the total gain for SBS (Table 4) invariant. This made it possible to reduce the two-photon contribution to the total absorption coefficient (Table 5) and to weaken the effect of the SBS phase mismatch due to the two-photon heating.

\subsection{Effects of Self-Focusing and Plasma Formation on Stimulated Scattering}

All light producing phenomena were inspected visually through a thin transparent glass wall of the cell.

First, consider the self-focusing. There are two mechanisms responsible for the self-focusing - the Kerr-effect and the electrostriction [31]. The relaxation time for the Kerr-effect is $\tau_{K} \approx 10^{-11} s$. The relaxation time for the electrostriction is approximately equal to the duration for the acoustic wave to pass through the focal caustic diameter. The minimal value for this time under our experimental conditions is

$$
\tau_{S T R}^{M I N} \approx \frac{F_{M I N} \theta}{v} \approx \frac{(11 \mathrm{~cm})\left(3 \times 10^{-4} \mathrm{rad}\right)}{10^{5} \mathrm{~cm} / \mathrm{s}} \approx 3 \times 10^{-8} \mathrm{~s}=30 \mathrm{~ns}
$$

which is considerably more than the pump pulse duration $\tau_{0} \approx 8 n s$. Therefore, as well as in [32, 33], under our experimental conditions only the Kerr-effect should be taken into account. The self-focusing is characterized by the critical power [34]

$$
P_{C R}=\frac{(1.22 \lambda)^{2} 4 \pi c}{128 c n n_{2}}=\frac{\pi(1.22 \lambda)^{2}}{32 n n_{2}} \approx 8 \times 10^{5} \mathrm{~W},
$$

where $n_{2} \approx 4 \times 10^{-13}[S G S] \approx 1.2 \times 10^{-12}\left[\mathrm{~cm}^{2} / \mathrm{kW}\right] \quad$ is a Kerr constant of hexane [26]. Our pump power is

$$
W_{L} \approx \frac{1.5 \mathrm{~mJ}}{8 \mathrm{~ns}} \approx 2 \times 10^{5} \mathrm{~W} \approx 0.25 P_{C R}
$$

For the input power $P<0.273 P_{C R}$ the self-focusing does not develop [34]. Hence, the self-focusing should not affect our experiments. Usually, the self-focusing in liquids is followed by the thin channels of light [35] and by increase in the divergence angle of the radiation passed through the cell in the forward direction. A visual inspection of the nonlinear liquid and the radiation did not detect these signs of the self-focusing.

Second, consider the plasma formation. The acoustical wave generated as a result of the SBS is supposed to be the reason for optical destruction in solids $[7,36]$. The dynamics of internal optical destruction in solids was studied in [37]. During the course of the laser pulse the destruction has the form of a thread placed along the focal caustic. The pace of the thread development is much higher than the speed of shock wave. The thread diameter is considerably smaller than the focal caustic diameter. Subsequent to the laser pulse the destruction grows in the transverse direction and possesses the form of discs. The SBS can not completely provide the energy transformation. Preference is given to the destruction mechanisms associated with the self-focusing and the multi-photon ionization.

The theory of the optical breakdown in liquids does not exist, because not much is known about it [1, 34]. For $\hbar \omega=4 \mathrm{eV}$ fast three-photon-ionization of hexane provides the initial electrons. The breakdown threshold depends on the electron multiplication rate. Different approaches (classic or quantum) are used to describe how the electron gains energy, depending on the relation between the photon energy $\hbar \omega$ and the average electron energy increment during a single collision $\Delta \varepsilon$ [38]. If $\Delta \varepsilon>\hbar \omega$, the classic kinetic equation can be used. If $\hbar \omega \geq \Delta \varepsilon$, the quantum kinetic equation containing the finite-difference expressions should be used. For our moderate pump intensity $I_{L}<10^{11} \mathrm{~W} / \mathrm{cm}^{2}$ we have $\Delta \varepsilon \approx 1 \mathrm{eV}$. Taking into account $\hbar \omega=4 \mathrm{eV}>\Delta \varepsilon \approx 1 \mathrm{eV}$ the quantum kinetic equation should be solved.

As a rough approximation, a liquid could be considered as a gas under ultrahigh pressure of thousands of atmospheres. For the noble gases under thousands of atmospheres [38, 39] the optical breakdown threshold reaches $E \approx 10^{6} \mathrm{~V} / \mathrm{cm}, I \approx(E / 20)^{2} \approx 2.5 \times 10^{9} \mathrm{~W} / \mathrm{cm}^{2}$. For the molecular gases [38, 40] the thresholds are hundred-fold higher than for the single-atom gases, because it is more difficult for the electron to pass the "dangerous" region between the exiting and the ionization.

Usually, the optical breakdown in liquid is followed by a flash of light [35] and bubbles. The visual inspection did not detect them. It is possible, that for the organic liquids like hexane the electromagnetic energy transmits to the hydrodynamic perturbations instead of the avalanche. Emergence of SBS and STS-2 in our experiments confirms this supposition.

\section{Conclusions}

The present study focuses on the stimulated scattering (SS) of nanosecond ( $5 \sim 10 \mathrm{~ns})$ near-UV ( $\lambda=193 \sim 351 \mathrm{~nm})$ laser pulses in liquids (hexane, heptane, and others). Some experimental results gained from the previous studies 
concerning the frequency shift and the phase-conjugation (PC) fidelity disagree with the existing theory of SS, which was developed for the near-IR $(\lambda=0.69 \sim 1.06 \mu \mathrm{m})$ spectral region and takes into account only the linear (single-photon) light absorption.

To resolve the inconsistency, $\mathrm{SS}$ of $\mathrm{XeCl}$ excimer laser radiation $(\lambda=308 \mathrm{~nm}$ ) with the duration $8 \mathrm{~ns}$ in liquid hexane for various values of the linear absorption coefficient $\alpha$ and the pump intensity $I_{L}$ is investigated experimentally.

In contrast to the previous near-UV studies, we managed to create an original master oscillator, which produced a single longitudinal mode. As it is well-known, such a single-mode regime is characterized not only by a short spectrum, but also by a near-Gaussian temporal behavior without the uncontrolled peaks of intensity (the mode interference). So our estimates of the pump intensity were much more reliable than the previous ones. Due to the smaller bandwidth we had less amplified beam energy and limited experimental possibilities as compared to the previous studies (we could not use the neutral filters to control the pump intensity). We also managed to create a spectrum analyzer, which monitored the temporal spectra of two counter-propagating beams in different but close enough fields. It allowed us to be sure that the right field involves only a pump spectrum, and the left field - only a backscattered spectrum. Without such analyzer, any slightly-shifted backscattered spectral line may be simply confused with a spectral line of the pump radiation.

When the product $\left(I_{L} L\right) \approx 1.5 \times 10^{3} \mathrm{MW} / \mathrm{cm}$ ( $L$ is the nonlinear interaction length) remains invariant, the experimental SS spectrum depends not only on $\alpha$, but also on $I_{L}$. For $\alpha<0.1 \mathrm{~cm}^{-1}$ and $I_{L} \geq 10^{10} \mathrm{~W} / \mathrm{cm}^{2}$, the spectrum includes a previously unknown line, corresponding to a previously unknown mechanism - the stimulated thermal scattering induced by the heating due to the two-photon absorption (two-photon STS-2). For $\alpha<0.1 \mathrm{~cm}^{-1}$ and $I_{L} \leq 10^{9} \mathrm{~W} / \mathrm{cm}^{2}$, the spectrum includes a previously unobserved line in the near-UV region, which corresponds to the genuine stimulated Brillouin scattering (SBS) and is characterized by the frequency shift $\Omega_{B}=0.33 \mathrm{~cm}^{-1}$, in full agreement with the theory. For $\alpha>0.1 \mathrm{~cm}^{-1}$ or $I_{L} \geq 10^{10} \mathrm{~W} / \mathrm{cm}^{2}$, SBS is suppressed by the phase mismatch due to the heating caused by the linear or two-photon absorption, respectively. To detect the genuine SBS line for $\alpha<0.1 \mathrm{~cm}^{-1}$, we had to reduce the pump intensity while keeping constant the total gain for SBS and thus to weaken the phase mismatch due to the two-photon heating.

When the two-photon STS-2 is used for the phase-conjugation, the PC fidelity is lower than that achieved by using the SBS because of the two-photon thermal phase self-modulation.

The molecular cross-section for the two-photon absorption of hexane at $\lambda=308 \mathrm{~nm} \quad \sigma_{2} \approx(2 \pm 1) \times 10^{-50} \mathrm{~cm}^{4} \mathrm{~s}$ is found from the experimental threshold pump intensity for the two-photon STS-2.

The "SBS" lines observed in the previous studies have frequency shifts much smaller than those predicted by the theory and must be interpreted as the two-photon STS-2 lines. The physical mechanisms of the linear STS-2 and the two-photon STS-2 are essentially different and their lines can readily be distinguished in the spectrum. The total inconsistency between the experimental behavior of the two-photon STS-2 and the linear STS-2 lines explains why the two-photon STS-2 lines observed in the previous studies were attributed to SBS for almost a decade.

The physical mechanism of the two-photon STS-2 is not specific for the near-UV spectral region.

\section{References}

[1] Y. R. Shen, The Principles of Nonlinear Optics. New York: Wiley, 1984 [Moscow: Nauka, 1989].

[2] S. Kielich, Molecular Nonlinear Optics. Warsaw: PWN, 1977 [Moscow: Nauka, 1981].

[3] V. G. Dmitriev, Nonlinear Optics and Phase Conjugation. Moscow: Fizmatlit, 2003 [in Russian].

[4] B. Ya. Zeldovich, N. F. Pilipetsky, and V. V. Shkunov, Principles of Phase Conjugation. Berlin: Springer, 1985 [Moscow: Nauka, 1985].

[5] I. L. Fabelinskii, Molecular Scattering of Light. New York: Plenum, 1968 [Moscow: Nauka, 1965].

[6] B. Ya. Zeldovich, V. I. Popovichev, V. V. Ragulskii, F. S. Faizullov, "Connection between the wave fronts of the reflected and exiting light in stimulated Mandel'shtam-Brrillouin scattering”, Pisma v Zh. Eksp. Teor. Fiz., vol. 15, pp. 160-163, 1972 [JETP Lett. 15, 109, 1972].

[7] R. Y. Chiao, C. H. Townes, B. P. Stoicheff, "Stimulated Brillooin scattering ang coherent generation of intensive hypersound waves", Phys. Rev. Lett. vol. 12, pp. 592-599, 1964.

[8] E. Garmire, C. H. Townes, "Stimulated Brillouin scattering in liquds”, Appl. Phys. Lett, vol.. 5, pp. 84-90, 1964.

[9] R. G. Brewer, K. E. Rieckhoff, "Stimulated Brillouin Scattering in liquids", Phys. Rev. Lett..vol. 13, pp. 334a-339, 1964.

[10] R. G. Brewer, "Ruby laser as amplifier of scattered Mandelstam=Brillouin radiation”, Appl. Phys. Lett., vol. 5, pp. 127-131, 1964.

[11] V.V. Kuzmin, "Phase synchronism breakdown for stimulated scattering", in Tr. Fiz. Inst. im P.N.Lebedeva, Acad. Nauk SSSR 207, pp. 3-39 (Nauka, Moscow, 1991) [Non-Linear Optics and Molecular Light Scattering, edited by N.G.Basov and I. L. Fabelinskii, Proc. P. N. Lebedev Phys. Inst., Vol. 207] [in Russian].

[12] S. F. Grigoriev, O. P. Zaskalko, and V. V. Kuzmin, "Specifics of SBS in absorbing media", Zh. Eksp. Teor. Fiz. 92, pp. 1246-1255, 1987 [JETP 65, 697 1987].

[13] V. S. Starunov, "Certain problems in the theory of the stimulated molecular scattering of light", Zh. Eksp. Teor. Fiz., vol. 57, pp. 1012-1018, 1969 [JETP 30, 553 1970]. 
[14] V. S. Starunov and I. L. Fabelinskii, "Stimulated Mandel'shtam-Brillouin scattering and stimulated entropy (temperature) scattering of light", Sov. Phys. Usp., vol. 12, pp. 463-489, 1970 [Usp. Fiz. Nauk, vol. 98, pp. 441-491, 1969].

[15] M. Statkine, I. J. Bijio, B. J. Feldman, and R. A. Fisher, "Efficient phase conjugation of an ultraviolet $\mathrm{XeF}$ laser beam by stimulated Brillouin scattering", Optics Letters, vol. 7, No. 3, pp. 108-110, 1982.

[16] M. C. Gower, "KrF laser amplifier with phase-conjugate Brillouin retroreflectors", Optics Letters, vol. 7, No. 9, pp. 423-425, 1982.

[17] M. C. Gower, "Phase conjugation at 193nm", Optics Letters, vol. 8, No. 2, pp. 70-72, 1983.

[18] E. Armandillo and D. Proch, "Highly efficient, high-quality phase-conjugate reflection at $308 \mathrm{~nm}$ using stimulated Brillouin scattering”, Optics Letters, vol. 8, No. 10, pp. 523-525, 1983.

[19] M. C. Gower and R.G.Caro,'KrF laser with a phase-conjugate Brillouin mirror", Optics Letters, vol. 7, No. 4, pp. 162-164, 1982.

[20] G. M. Davis and M. C. Gower, "Stimulated Brillouin scattering of a KrF laser", IEEE J. Quant. Electron., vol. 27, No. 3, pp. 496-501, 1991.

[21] S. S. Alimpiev, V. S. Bukreev, S. K. Vartapetov, I. A. Veselovskii, V. S. Nersisian, A. Z. Obidin, and A. M. Prokhorov, "Line narrowing and wavefront reversal of radiation of an XeCl laser", Sov. Phys.-Lebedev Inst. Reports, No. 12, pp. 12-15, 1989 [Kratk. Soobshch. Fiz., No. 12, pp. 11-13, 1989].

[22] S. S. Alimpiev, V. S. Bukreev, S. K. Vartapetov, I. A. Veselovskii, B. I. Kusakin, S. V. Lihanckii, and A. Z. Obidin, "Line narrowing and wavefront reversal of radiation of an $\mathrm{KrF}$ laser", Quant. Electron., vol. 21, pp.80-81, 1991 [Kvant. Elektron. (Moscow), vol. 18, pp. 89-90, 1991].

[23] A. L. Egorov, V. V. Korobkin, and R. V. Serov, "Single mode Nd-glass laser", Kvant. Elektron. (Moscow) voll, 2, pp. 513-518, 1975 [Quant. Electron. 5, 291 1975].

[24] A. J. Alcock, C. De. Michelis, M. C. Richardson, "Breakdown and selffocusing effects in gases produced by means of single mode ruby laser", IEEE J. Quant. Electron., vol. 6, No. 10, pp. 622-629, 1970.

[25] V. F. Nozdrev, The Use of Ultrasonics in Molecular Physics. Oxford: Pergamon, 1965 [Moscow: Fizmatlit, 1958].

[26] P. P. Ho and R. R. Alfano, "Optical Kerr effect in liquids", Phys. Rev. A., vol. 20, No. 5. pp.. 2170-2182, 1979.
[27] A. J. Gordon and R. A. Ford, The Chemist's Companion. New York: Wiley, 1972

[28] Yu. N. Karamzin, A. P. Sukhorukov, and V. A. Trofimov, Mathematical Modeling in Nonlinear Optics. Moscow: State University, 1989 [in Russian].

[29] M. Ammosov, N. Delone, M. Ivanov, I. Bondar and A. Masalov, Adv. in Atom., Mol. and Opt. Phys., vol. 29, pp. 34-40, 1992.

[30] N. B. Delone and V. P. Krainov, Fundamentals of Nonlinear Optics of Atomic Gases. New York: Wiley, 1988 [Moscow: Nauka, 1986].

[31] R. Y. Chiao, E. Garmire, C. H. Townes, "Selffocusing of optical mazer beam", Phys. Rev. Lett. Vol. 13, pp.479-487, 1964.

[32] Y. R. Shen, "Electrostriction, optical Kerr effect and selffocusing of laser beams", Phys. Lett. Vol. 20, pp. 378-384, 1966.

[33] P. Lallemand, N. Bloembergen, "Selfocusing of laser beams and stimulated Raman scattering", Phys. Rev. Lett. vol. 15, pp. $1010=1016,1965$.

[34] J. F. Reintjes, Nonlinear Optical Parametric Processes in Liquids and Gases. New York, London, Tokyo: Academic Press, 1984.[ Moscow: Mir, 1987].

[35] R. G. Brewer, J. R. Lifsits, "Narrow optical channels and instabilities in liquid under laser beam", Phys. Lett. vol. 23, pp. 79-84, 1966.

[36] C. R. Guiliano, "Destruction of transparent dielectric materials under laser radiation", Appl. Phys. Lett., vol. 5, pp, 137-143, 1964.

[37] J. P. Budin, J. Raffy, "Dynamics of destruction in glasses caused by laser radiation", Appl. Phys. Lett. vol. 9, pp, 291-296, 1966

[38] Yu. P. Raizer, Laser-Induced Discharge Phenomena.. New York: Consultants Bureau, 1977 [Moscow: Nauka, 1974;].

[39] D. H. Gill, A. A. Dougal, "Minimums of threshold curves for laser breakdown in gases under high pressure caused by ionization by electron impact”, Phys. Rev. Lett., vol. 15, pp. 845-850, 1965.

[40] Yu. B. Afanasiev, Ye. M. Belenov, I. A. Poluektov, "Optical breakdown of molecular gases", Pisma v Zh. Eksp. Teor. Fiz., vol. 15, pp. 60=63, 1972 [JETP Lett. 15, 41 1972]. 